\title{
Effects of dexmedetomidine postconditioning on myocardial ischemia and the role of the PI3K/Akt-dependent signaling pathway in reperfusion injury
}

\author{
XIANG YANG CHENG ${ }^{1,2}$, XIAO YU GU ${ }^{2,3}$, QIN GAO $^{3,4}$, QIAO FENG ZONG ${ }^{3}$, XIAO HONG LI $^{2}$ and YE ZHANG ${ }^{1}$ \\ ${ }^{1}$ Department of Anesthesiology, The Second Affiliated Hospital of Anhui Medical University, Hefei, Anhui 230601; \\ ${ }^{2}$ Department of Anesthesiology, The First Affiliated Hospital of Bengbu Medical College, \\ Bengbu, Anhui 233004; ${ }^{3}$ Department of Physiology; ${ }^{4}$ Anhui Key Laboratory of Tissue Transplantation, \\ Bengbu Medical College, Bengbu, Anhui 233030, P.R. China
}

Received January 24, 2016; Accepted May 13, 2016

DOI: $10.3892 / \mathrm{mmr} .2016 .5345$

\begin{abstract}
The present study aimed to determine whether post-ischemic treatment with dexmedetomidine (DEX) protected the heart against acute myocardial ischemia/reperfusion (I/R)-induced injury in rats. The phosphatidylinositol-3 kinase/protein kinase $\mathrm{B}(\mathrm{PI} 3 \mathrm{~K} / \mathrm{Akt})$-dependent signaling pathway was also investigated. Male Sprague Dawley rats $(n=64)$ were subjected to ligation of the left anterior descending artery (LAD), which produced ischemia for $25 \mathrm{~min}$, followed by reperfusion. Following LAD ligation, rats were treated with $\operatorname{DEX}(5,10$ and $20 \mu \mathrm{g} / \mathrm{kg})$ or underwent post-ischemic conditioning, which included three cycles of ischemic insult. In order to determine the role of the PI3K/Akt signaling pathway, wortmannin (Wort), a PI3K inhibitor, was used to treat a group of rats that had also been treated with DEX $(20 \mu \mathrm{g} / \mathrm{kg})$. Post-reperfusion, lactate dehydrogenase (LDH), cardiac troponin I (cTnI), creatine kinase isoenzymes (CK-MB), superoxide dismutase (SOD) and malondialdehyde (MDA) serum levels were measured using an ultraviolet spectrophotometer. The protein expression levels of phosphorylated (p)-Akt, Ser9-p-glycogen synthase kinase-3 $\beta$ (p-GSK-3 $\beta$ ) and cleaved caspase- 3 were detected in heart tissue by western blotting. The mRNA expression levels of B-cell lymphoma 2 (Bcl-2) and Bcl-2-associated X protein (Bax) were detected using reverse transcription-polymerase chain reaction. At the end of the experiment, the hearts were removed and perfused in an isolated perfusion heart apparatus with Evans blue (1\%) in order to determine the non-ischemic
\end{abstract}

Correspondence to: Professor Ye Zhang, Department of Anesthesiology, The Second Affiliated Hospital of Anhui Medical University, 678 Fu Rong Road, Hefei, Anhui 230601, P.R. China E-mail: zhangye_hassan@aliyun.com

Key words: dexmedetomidine, cardioprotection, apoptosis, ischemia/reperfusion, phosphatidylinositol-3 kinase/protein kinase B signaling pathway areas. The risk and infarct areas of the heart were not dyed. As expected, I/R induced myocardial infarction, as determined by the increased serum levels of cTnI, CK-MB and MDA, and the decreased levels of SOD. Post-ischemic treatment with DEX increased the expression levels of p-Akt and p-GSK-3 $\beta$, whereas caspase-3 expression was reduced following DEX treatment compared with in the I/R group. Compared with the I/R group, the ratio of $\mathrm{Bcl}-2 / \mathrm{Bax}$ at the mRNA level was elevated in the DEX and ischemic post-conditioning groups, whereas the expression levels of Bax were decreased. Conversely, the effects of DEX were attenuated by Wort. These results indicated that, similar to post-ischemic conditioning, post-ischemic treatment with DEX protects the heart against I/R via the PI3K/Akt-dependent signaling pathway, possibly by activating GSK-3 $\beta$.

\section{Introduction}

The incidence of acute myocardial infarction (MI) has exhibited an increasing trend. The most effective therapeutic measure against myocardial ischemia is the restoration of myocardial perfusion; however, myocardial ischemia/reperfusion (I/R) injury is a serious consequence. Myocardial I/R may induce excessive reactive oxygen species to attack cells in the reperfused area. Ultrastructural damage to the myocardium is irreversible, and can lead to disordered metabolism and function.

Dexmedetomidine (DEX) is a novel $\alpha-2$ agonist with sedative properties. DEX has been used as a sedative in numerous settings, increasing the cooperation of patients without depressing their respiration $(1,2)$. DEX has high potential for use in clinical anesthesia, and due to its wide effect on inflammatory responses, may have a protective role in myocardial I/R (3). It has previously been reported that DEX pre-treatment improves myocardial I/R injury in isolated hearts (4); however, whether DEX post-treatment can protect the heart from $\mathrm{I} / \mathrm{R}$ injury in vivo remains unknown (5).

Phosphatidylinositol-3 kinase/protein kinase B (PI3K/Akt) is anintracellularsignaling pathway, which has diverse biological 
Table I. Reverse transcription-polymerase chain reaction primers for $\mathrm{Bax}, \mathrm{Bcl}-2$ and $\beta$-actin.

\begin{tabular}{llll}
\hline Gene & Primer & Sequence & Product (bp) \\
\hline Bax & Forward & 5'-GGATCGAGCAGAGAGGATGG-3' \\
& Reverse & 5'-TGGTGAGTGAGGCAGTGAGG-3' & 464 \\
Bcl-2 & Forward & 5'-CTGGTGGACAACATCGCTCTG-3' \\
& Reverse & 5'-GGTCTGCTGACCTCACTTGTG-3' & 227 \\
& Forward & 5'-GATGGTGGGTATGGGTCAGAAGGAC-3' & 630 \\
\hline
\end{tabular}

Bcl-2, B-cell lymphoma 2; Bax, Bcl-2-associated X protein.

actions, and is involved in cell survival, apoptosis, growth, energy metabolism and migration (6). Akt, also known as $\mathrm{PKB}$, is a downstream target of PI3K; when Akt is activated by upstream signaling factors (PI3K-phosphoinositide-dependent kinase-1), it can activate glycogen synthase kinase (GSK)-3 $\beta$ to phosphorylated (p)-GSK-3 $\beta$, which is believed to be the integration point of several pathways and has an important role in cardioprotection (7). In addition, it has been hypothesized that GSK-3 $\beta$ is a functional downstream target of Akt (7). Cysteine aspartate protease-3 (caspase-3) is one of the most important enzymes in the caspase family, and is involved in the apoptotic process following activation by other members of the caspase family. Caspase-3 is a significant protease in the cascade reaction, and is therefore known as the 'hallmark enzyme' of cell apoptosis (8). Cleaved caspase-3 is the activated form of caspase-3. Therefore, when Akt is activated, I/R-induced myocardial injury may be reduced, via the involvement of GSK-3 $\beta$, the B-cell lymphoma 2 (Bcl-2) family and caspase-3.

The present study used an in vivo $\mathrm{I} / \mathrm{R}$ rat model to determine whether post-ischemic treatment with DEX exerted protective effects against I/R-induced MI. Lactate dehydrogenase (LDH), cardiac troponin I (cTnI), creatine kinase isoenzymes (CK-MB), superoxide dismutase (SOD) and malondialdehyde (MDA) serum levels were measured, as well as the infarct size of the heart. The most adverse effect of myocardial I/R injury is irreversible necrosis. Myocardial enzyme serum levels are one of the most important indexes used to determine the degree of myocardial necrosis (9). LDH, CK-MB and cTnI are markers of myocardial injury (10-12). CTnI is only expressed in the myocardium, and is therefore a specific marker of myocardial injury and is considered the 'gold standard (13)' for the identification of myocardial injury. MDA is commonly used as a biomarker of lipid peroxidation, whereas SOD is an antioxidant enzyme that protects tissues against oxidative stress. All of these parameters indicate the severity of myocardial ischemia.

In order to investigate the underlying mechanisms, the present study measured the expression levels of apoptosis-associated genes and proteins, and evaluated alterations to the PI3K/Akt signal transduction pathway. Bcl-2 was the first gene to be identified as an inhibitor of apoptosis, which has been reported to work as a downstream target of GSK-3 $\beta$ (7). When the expression levels of Bax are increased, Bax can form homodimers with Bcl-2, and proapoptotic factors, such as

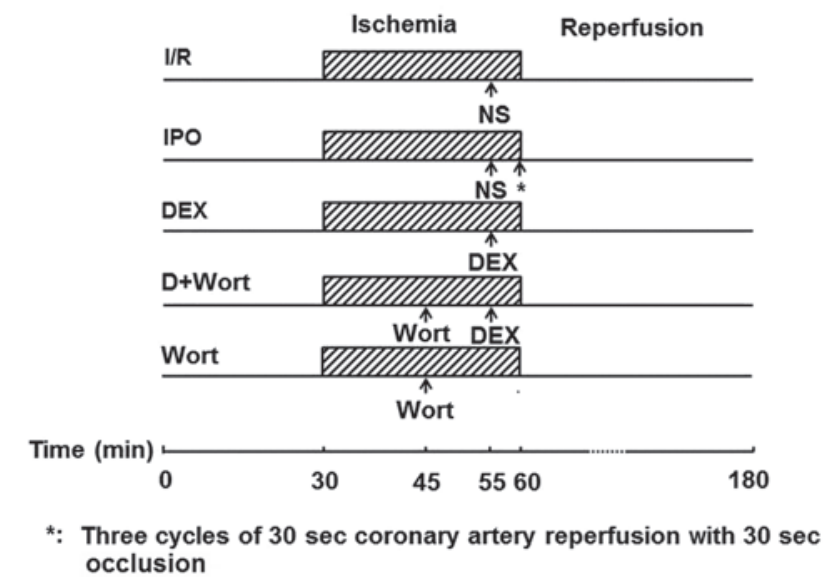

Figure 1. Experimental protocols used for in vivo experiments. I/R, ischemia/reperfusion group; IPO, ischemic post-conditioning group; D+Wort, DEX + Wort group; NS, normal saline; DEX, dexmedetomidine; Wort, wortmannin.

cytochrome $c$ can be released, eventually activating the downstream factor caspase-3 and promoting apoptosis. Conversely, Bcl-2 exerts an anti-apoptotic role via its effects on Bax; therefore, the ratio of $\mathrm{Bcl}-2 / \mathrm{Bax}(13,14)$ regulates apoptosis to some degree.

The results of the present study indicated that post-ischemic treatment with DEX exerted protective effects against I/R-induced MI via the PI3K/Akt signal transduction pathway. These effects were very likely induced via $\mathrm{p}-\mathrm{GSK}-3 \beta$. Further studies are required to delineate the effects of DEX with $\alpha-2$ adrenergic receptor stimulation.

\section{Materials and methods}

Reagents. DEX was purchased from Jiangsu Nhwa Pharmaceutical Corporation Ltd. (Jiangsu, China). Wortmannin (Wort) was obtained from Sigma-Aldrich (St. Louis, MO, USA). RatCK-MB isoenzyme enzyme linked-immunosorbent assay (ELISA) kit (cat. no. E006), LDH assay kit cat. no. A020-2), cTnI ELISA kit (cat. no. H149-2), SOD assay kit (cat. no. A001-3) and MDA assay kit (cat. no. A003-1) were obtained from Jiancheng Institute of Biotechnology (Nanjing, China). TRIzol ${ }^{\circledR}$ reagent was purchased from Invitrogen (Thermo Fisher Scientific, Inc., Waltham, MA, USA). Bcl-2, Bax and $\beta$-actin primers were acquired from Sangon Biotech 
A

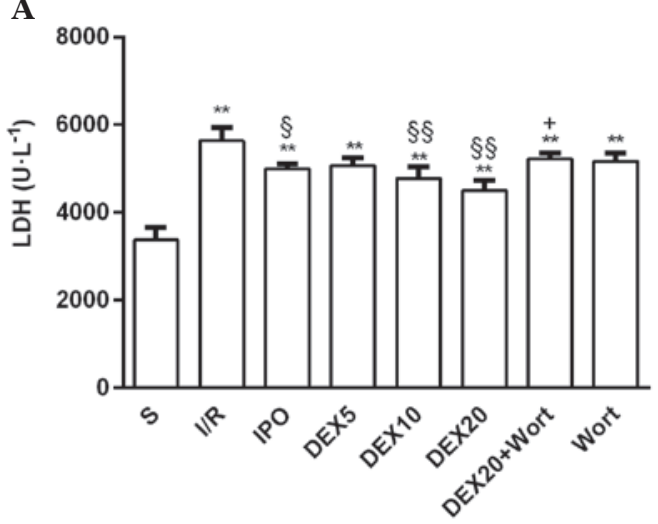

C

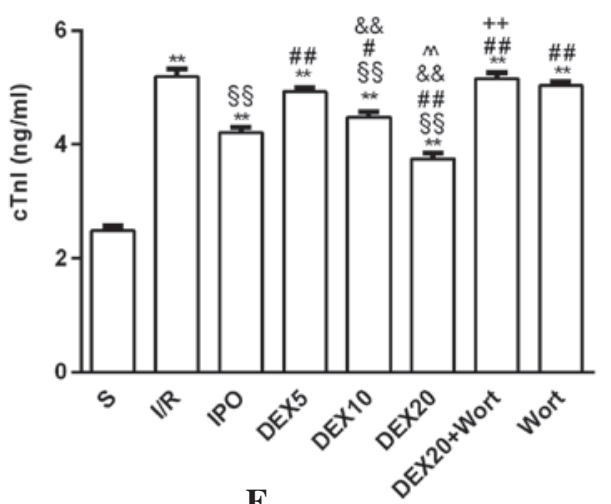

B

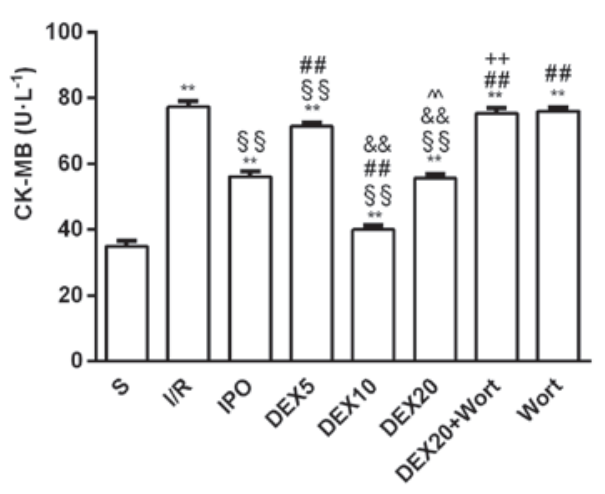

D

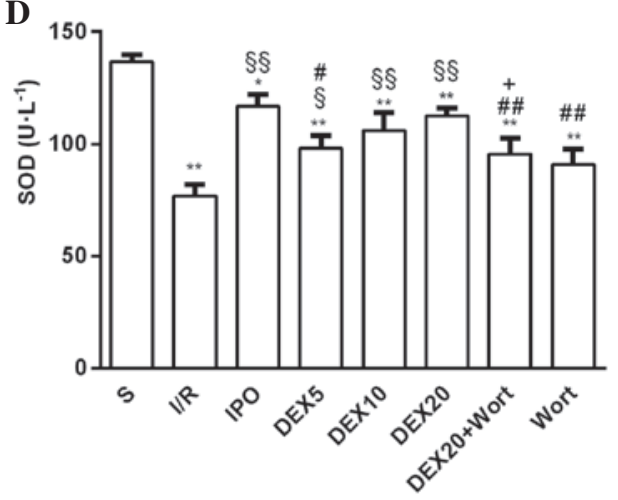

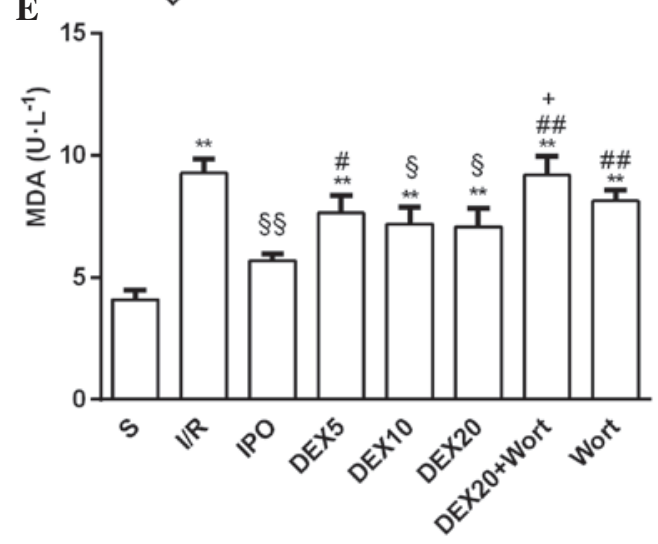

Figure 2. (A) LDH, (B) CK-MB, (C) cTnI, (D) SOD and (E) MDA levels in the serum of rats from each group. Data are presented as the mean \pm standard error of the mean from at least six independent experiments. ${ }^{*} \mathrm{P}<0.05,{ }^{* *} \mathrm{P}<0.01$ vs. $\mathrm{S}$ group, ${ }^{\S} \mathrm{P}<0.05,{ }^{\S \S} \mathrm{P}<0.01 \mathrm{vs}$. I/R group; ${ }^{\#} \mathrm{P}<0.05,{ }^{\# \#} \mathrm{P}<0.01$ vs. IPO group, ${ }^{\& \&} \mathrm{P}<0.01$ vs. DEX5 group; ${ }^{\wedge} \mathrm{P}<0.01$ vs. DEX10 group; ${ }^{+} \mathrm{P}<0.05,{ }^{+} \mathrm{P}<0.01$ vs. DEX20 group. $\mathrm{LDH}$, lactate dehydrogenase; cTnI, cardiac troponin I; CK-MB, creatine kinase isoenzymes; MDA, malondialdehyde; SOD, superoxide dismutase; S, sham; I/R, ischemia/reperfusion; IPO, ischemic post-conditioning; DEX, dexmedetomidine; Wort, wortmannin.

Co., Ltd. (Shanghai, China). The following primary antibodies were obtained from Cell Signaling Technology, Inc. (Danvers MA, USA): Mouse anti-GSK-3 $\beta$ (1:2,000 dilution; cat. no. BF0695), rabbit polyclonal anti-p-GSK-3 $\beta(1: 2,000$ dilution; cat. no. AF2016), rabbit polyclonal anti-Akt (1:1,000 dilution; cat. no. 9272S), rabbit polyclonal anti-p-Akt (1:1,000 dilution; cat. no. 4060S) and rabbit polyclonal anti-cleaved caspase-3 (1:2,000 dilution; cat. no. 9661S). Horseradish peroxidase (HRP)-linked anti-mouse immunoglobulin ( $\operatorname{IgG}$ ) (1:10,000 dilution; cat. no. BA1050), HRP-linked anti-rabbit IgG (1:10,000 dilution; cat. no. BA1054) and anti- $\beta$-actin (1:1,000 dilution; cat. no. BM0627) were acquired from Wuhan Boster Biological Technology Co., Ltd. (Wuhan, China).
Experimental animals. Male Sprague Dawley (SD) rats (weight, 250-300 g) were purchased from the Experimental Animal Center of Bengbu Medical Collage (Bengbu, China). All of the rats had ad libitum access to food and water, were maintained in a temperature and humidity-controlled environment $\left(22-26^{\circ} \mathrm{C} ; 50 \%\right.$ humidity) with a 12 hours light/dark cycle, and were raised in plastic cages. All animal procedures were conducted in accordance with the United States National Institutes of Health Guide and were approved by the Animal Use and Care Committee of Bengbu Medical College.

Animal preparation and experimental design. A total of 64 male SD rats were randomly assigned to the following eight groups: Group 1, sham group ( $\mathrm{S} ; \mathrm{n}=8$ ), the left anterior 
descending artery (LAD) was threaded but not ligated for $150 \mathrm{~min}$; group $2, \mathrm{I} / \mathrm{R}$ group $(\mathrm{n}=8)$, the rats were injected with $0.5 \mathrm{ml}$ normal saline (NS) after $25 \mathrm{~min}$ of LAD ligation, following $30 \mathrm{~min}$ of LAD ligation the rats underwent reperfusion for $120 \mathrm{~min}$; group 3, ischemic post-conditioning group (IPO; $\mathrm{n}=8$ ), the rats received $0.5 \mathrm{ml}$ normal saline (NS) after 25 min of LAD ligation, following 30 min of LAD ligation the hearts were subjected to three cycles of $30 \mathrm{sec}$ coronary artery reperfusion with $30 \mathrm{sec}$ occlusion, followed by $120 \mathrm{~min}$ of reperfusion; groups 4-6, DEX5, DEX10 (15) and DEX20 groups ( $\mathrm{n}=8 /$ group), the rats were intravenously injected with 5,10 or $20 \mu \mathrm{g} / \mathrm{kg}$ DEX after $25 \mathrm{~min}$ of LAD ligation, respectively, following $30 \mathrm{~min}$ of LAD ligation the rats underwent reperfusion for $120 \mathrm{~min}$; group 7, DEX20 + Wort group $(n=8)$, the rats were intravenously injected with $15 \mu \mathrm{g} / \mathrm{kg}$ Wort (16) $15 \mathrm{~min}$ after LAD ligation and were then injected with $20 \mu \mathrm{g} / \mathrm{kg}$ DEX 5 min prior to $120 \mathrm{~min}$ reperfusion; group 8 , Wort group $(\mathrm{n}=8)$, the rats were intravenously injected with $15 \mu \mathrm{g} / \mathrm{kg}$ Wort after $15 \mathrm{~min}$ of LAD ligation, following $30 \mathrm{~min}$ of LAD ligation the rats underwent reperfusion for $120 \mathrm{~min}$ (Fig. 1).

Establishment of a myocardial I/R model. The rats were anesthetized with $4 \%$ chloral hydrate $(1 \mathrm{ml} / 100 \mathrm{~g}$ body weight; i.p.), and in order to maintain anesthesia, $0.5 \mathrm{ml} 4 \%$ chloral hydrate was intraperitoneally injected periodically $(0.5 \mathrm{ml} / 100 \mathrm{~g}$ body weight). Following tracheal intubation, ventilation was provided via respiratory equipment, at a respiratory rate of $70-80$ times/min and a tidal volume of $2-3 \mathrm{ml} / 100 \mathrm{~g}$. Standard electrocardiograms (ECG) were recorded. Using the Medlab biological signal collecting and processing system (Nanjing Mei Yi Technology Co., Ltd., Nanjing, China), hemodynamic parameters were continuously measured by catheterization of the left common carotid artery. A left parasternal incision was made through the third and fourth ribs, and the pericardium was gently opened to expose the heart. The LAD was ligated with a 5-0 silk suture $(2 \mathrm{~mm})$ below the left atrial appendage and the left edge of the pulmonary cone. Its circumflex branch and the suture ends were threaded through a polyethylene tube to form snares for reversible coronary artery occlusion. Myocardial ischemia was induced via compression of the LAD by tightening the silk suture around the polyethylene tube following $20 \mathrm{~min}$ stabilization. An elevated ST-segment in the ECG indicated the success of ischemia. After 30 min of ischemia, the polyethylene tubes were loosened for $120 \mathrm{~min}$ to mimic reperfusion (17).

Measurement of serum LDH, $c T n I, C K-M B, S O D$ and MDA levels. At the end of reperfusion, arterial blood samples were collected, placed in test tubes with heparin, and centrifuged at $3,000 \mathrm{x}$ g for $15 \mathrm{~min}$ at $4^{\circ} \mathrm{C}$. The perfusion fluid was collected and stored at $-80^{\circ} \mathrm{C}$, and was thawed once prior to analysis. LDH, cTnI, CK-MB and MDA contents, and SOD activity were measured using commercially available kits according to the manufacturer's protocols.

Assessment of myocardial infarct size. At the end of reperfusion, heart tissues were collected from rats that had been anesthetized $4 \%$ chloral hydrate $(1 \mathrm{ml} / 100 \mathrm{~g}$ body weight; i.p.) and the excess blood was removed using Krebs-Henseleit

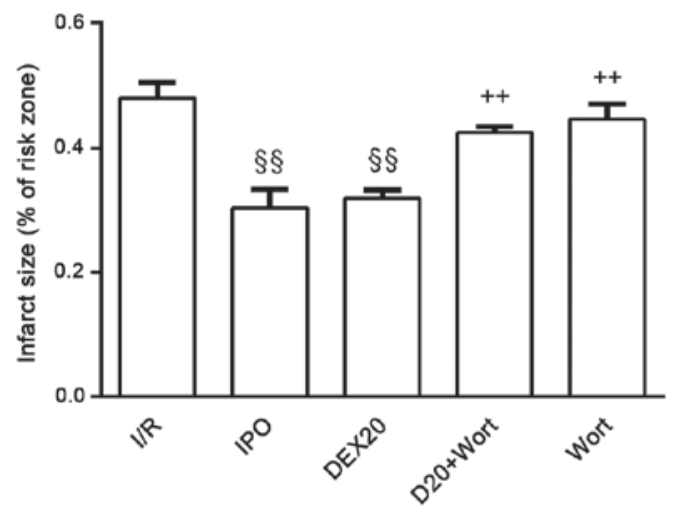

Figure 3. Comparison of infarct size in each experimental group in vivo. Data are presented as the mean \pm standard error of the mean from at least four independent experiments. ${ }^{\S \S} \mathrm{P}<0.01$ vs. I/R group; ${ }^{++} \mathrm{P}<0.01$ vs. DEX20 group. $\mathrm{I} / \mathrm{R}$, ischemia/reperfusion; IPO, ischemic post-conditioning; DEX, dexmedetomidine; Wort, wortmannin.

saline. Following re-occlusion of the left coronary artery in isolated Langendorff-perfused equipment, the hearts were perfused with $1 \%$ Evans blue dye, in order to delineate the non-ischemic area, since the risk and infarct areas remained undyed. The tissues were then frozen at $-20^{\circ} \mathrm{C}$ for several hours, prior to being sliced transversely into $2-3 \mathrm{~mm}$ sections. The slices were subsequently incubated in $1 \%$ tetrazolium chloride buffer solution ( $\mathrm{pH} \mathrm{7.4)}$ for $10-15 \mathrm{~min}$ at $37^{\circ} \mathrm{C}$, and were fixed in $10 \%$ buffered formalin. Infarct (pale) and risk (red) areas were calculated by planimetry using Image-Pro Plus software (version 6.0; Media Cybernetics, Inc., Rockville, MD, USA). Infarct size was expressed as the percentage of risk area.

Western blot analysis of Akt, p-Akt, cleaved caspase-3, $G S K-3 \beta$ and $p-G S K-3 \beta$. Western bot analysis was performed using proteins extracted from heart tissue samples. The DEX20 group was chosen as the treatment group used to investigate the underlying mechanisms of DEX post-conditioning, since the synthetic protective effects were more obvious in the DEX20 group compared with the DEX5 and DEX10 groups, according to serum enzyme level alterations. Western blot analyses were performed as previously described (17).

Reverse transcription-polymerase chain reaction (RT-PCR) analysis of Bax and Bcl-2 mRNA expression. Gene expression analysis was performed via RT-PCR using RNA from the heart tissue samples, as previously described (16). The primer sequences are presented in Table I.

Statistical analysis. Data are presented as the mean \pm standard error of the mean. Differences between treatment groups were assessed by one-way analysis of variance and Student-Newman-Keuls multiple comparison test using SPSS 16.0 software (SPSS Inc., Chicago, IL, USA). P $<0.05$ was considered to indicate a statistically significant difference.

\section{Results}

Alterations to the serum $L D H, C K-M B, c T n I, S O D$ and MDA levels in each group. Compared with the S group, LDH, 
A

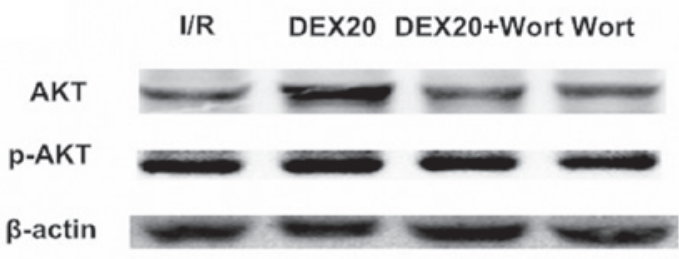

C

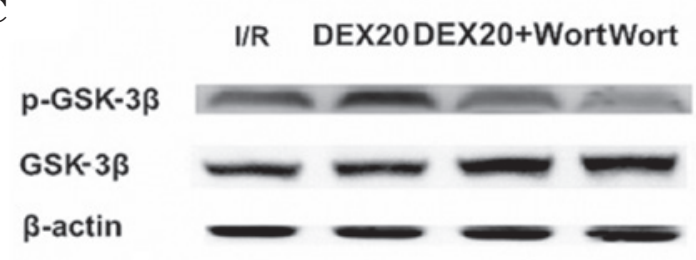

B

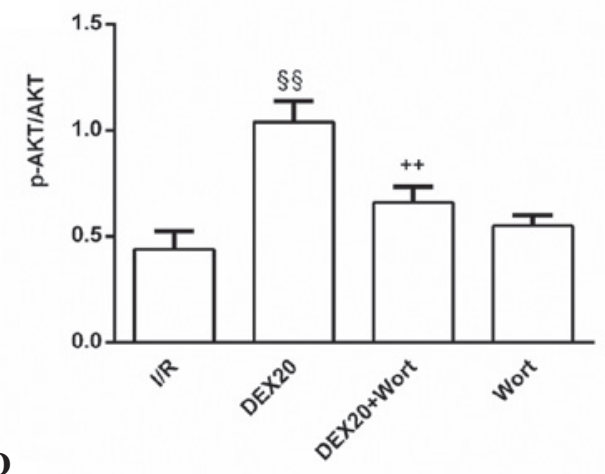

D

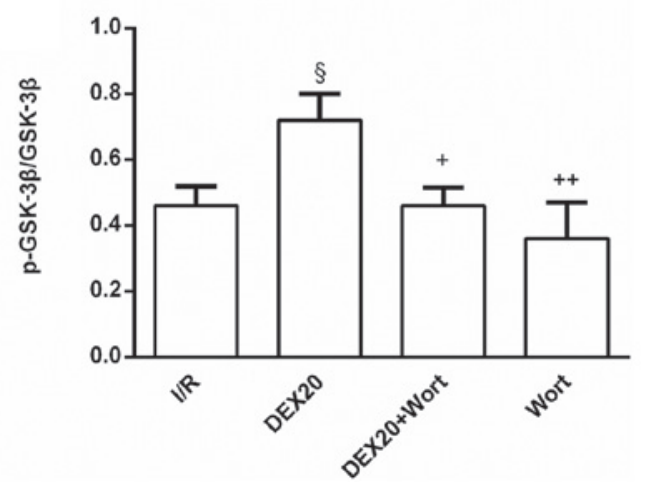

Figure 4. Expression levels of (A and B) p-Akt and (C and D) p-GSK-3 $\beta$ (Ser9) in heart tissue. Representive western blots of (A) p-Akt and (C) p-GSK-3 $\beta$ (Ser9) in the heart of each experimental group. $\beta$-actin was used as a loading control. (B and D) Average quantification obtained by densitometric analysis of the results of western blot analysis. Data are presented as the mean \pm standard error of the mean from at least four independent experiments. ${ }^{\S} \mathrm{P}<0.05,{ }^{\$ \S} \mathrm{P}<0.01 \mathrm{vs}$. I/R group; ${ }^{+} \mathrm{P}<0.05,{ }^{+} \mathrm{P}<0.01$ vs. DEX20 group. p-, phosphorylated; Akt, protein kinase B; GSK-3 $\beta$, glycogen synthase kinase-3 $\beta$; I/R, ischemia/reperfusion; DEX, dexmedetomidine; Wort, wortmannin.

cTnI, CK-MB and MDA levels in all of the other groups were increased, whereas SOD activity was decreased. Compared with the I/R group, the activities of LDH and CK-MB, and cTnI and MDA levels were reduced in the IPO, DEX10 and DEX20 groups; however, SOD activity was elevated. In addition, LDH, cTnI, CK-MB and MDA levels were significantly increased in the DEX20 + Wort group compared with the DEX20 group, whereas SOD activity was decreased (Fig. 2).

Effects on myocardial infarct size in each group. Compared with the I/R group, infarct size in the IPO and DEX20 groups was decreased. Compared with the DEX20 group, infarct size in the DEX20 + Wort group was markedly increased (Fig. 3).

Alterations to the protein expression levels of $p$-Akt and $p-G S K-3 \beta$. The protein expression levels of $\mathrm{p}$-Akt and p-GSK-3 $\beta$ in the DEX20 group were increased compared with those in the I/R group. Conversely, p-Akt and p-GSK-3 $\beta$ levels in the DEX20 + Wort group were lower than in the DEX20 group (Fig. 4).

Alterations to the protein expression levels of cleaved caspase-3. A significant decrease in the expression levels of cleaved caspase-3 was detected in the DEX20 group compared with in the I/R group. However, in the DEX20 + Wort group, a significant increase in cleaved caspase- 3 was detected, as compared with in the DEX20 group. The protein expression levels for each sample were determined as a percentage of the corresponding $\beta$-actin levels (Fig. 5).

Alterations to the mRNA expression levels of Bcl-2 and Bax. A marked increase in the expression levels of Bcl-2 and the ratio of Bcl-2/Bax were detected in the DEX20 group compared with the I/R group, whereas Bax expression was decreased. In the DEX20 + Wort group, Bcl-2 levels and the ratio of Bcl-2/Bax were significantly decreased compared with the DEX20 group, whereas Bax expression was increased (Fig. 6).

\section{Discussion}

The present study demonstrated that post-ischemic treatment with DEX, a novel $\alpha-2$ agonist with sedative properties, significantly attenuated the increased levels of LDH, cTnI, CK-MB and MDA, and the reduced activity of SOD induced by I/R, similar to post-ischemic preconditioning. In addition, DEX reduced infarct size. These observations indicated that DEX may exert cardioprotective effects.

Notably, the present study revealed that DEX increased the expression levels of $\mathrm{p}$-Akt and p-GSK $3 \beta$ in myocardium. In addition, DEX decreased the expression levels of the downstream proteins of the PI3K/Akt signaling pathway, Bax and cleaved caspase-3, and increased the expression levels of Bcl-2 and the ratio of $\mathrm{Bcl}-2 / \mathrm{Bax}$. Conversely, the effects of DEX on I/R-induced myocardial injury and PI3K/Akt signaling were attenuated by Wort, a noncompetitive inhibitor of PI3Ks. 
A

\section{I/R DEX20 DEX20+Wort Wort}

Cleaved caspase-3

$\beta$-actin

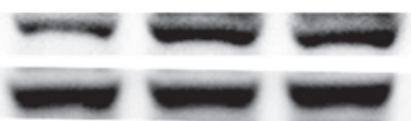

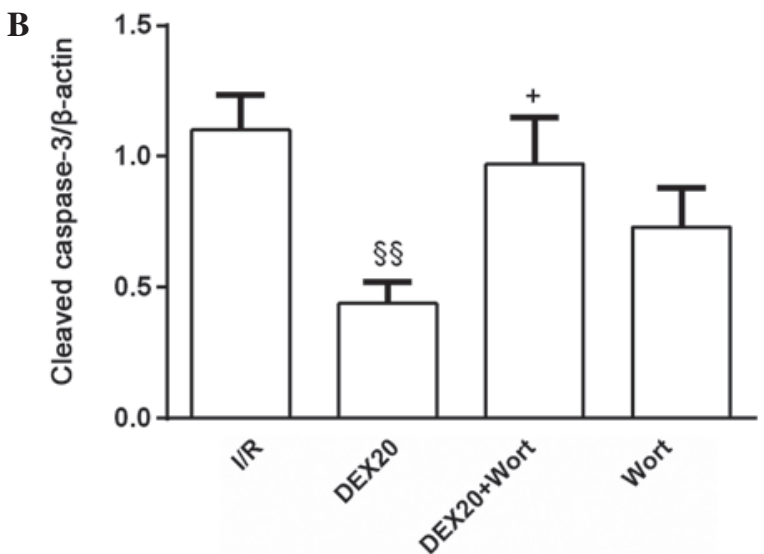

Figure 5. Expression levels of cleaved caspase-3 in heart tissue of each group. (A) Western blotting of cleaved caspase-3. $\beta$-actin was used as a loading control (B) Average quantification obtained by densitometric analysis of the results of western blot analysis. Data are presented as the mean \pm standard error of the mean from at least four independent experiments. ${ }^{\S} \mathrm{P}<0.01 \mathrm{vs}$. I/R group; ${ }^{+} \mathrm{P}<0.05 \mathrm{vs}$. DEX20 group. I/R, ischemia/reperfusion; DEX, dexmedetomidine; Wort, wortmannin.
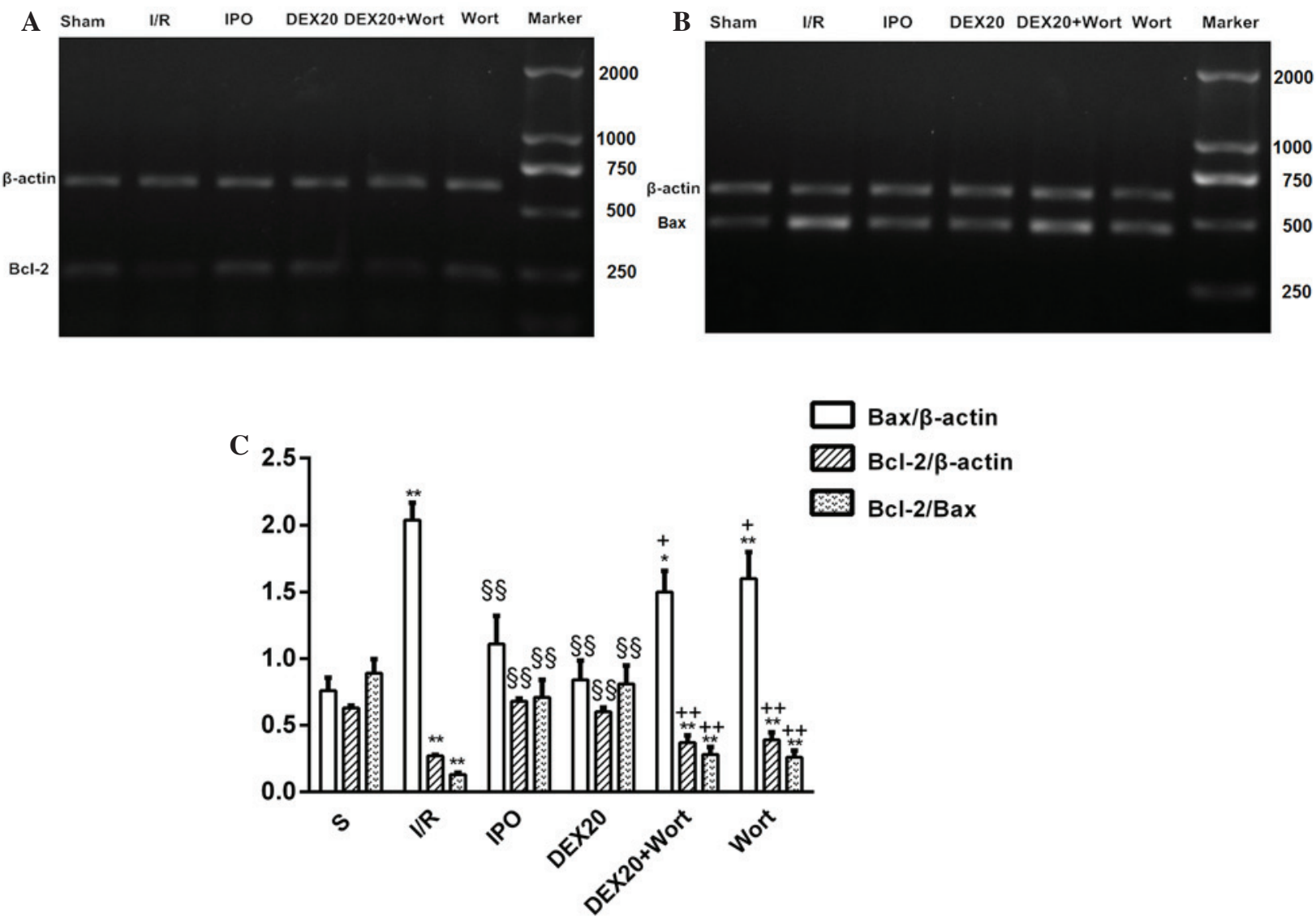

Figure 6. mRNA expression levels of myocardial Bcl-2 and Bax, and quantification of the Bcl-2/Bax ratio in the various groups. (A and B) Results of RT-PCR analysis in the heart tissue. $\beta$-actin was used as a loading control. (C) Quantification of the Bcl-2/Bax ratio obtained by densitometric analysis of RT-PCR. Data are presented as the mean \pm standard error of the mean from at least six independent experiments. ${ }^{*} \mathrm{P}<0.05,{ }^{* * *} \mathrm{P}<0.01$ vs. $\mathrm{S}$ group; ${ }^{\$ \$} \mathrm{P}<0.01 \mathrm{vs}$. I/R group; ${ }^{+} \mathrm{P}<0.05,{ }^{++} \mathrm{P}<0.01$ vs. DEX20 group. Bcl-2, B-cell lymphoma 2; Bax, Bcl-2-associated X protein; RT-PCR, reverse transcription-polymerase chain reaction; S, sham; I/R, ischemia/reperfusion; IPO, ischemic post-conditioning; DEX, dexmedetomidine; Wort, wortmannin.

These results indicated that DEX may have an important role in myocardial protection by activating the PI3K/Akt signaling pathway possibly via activation of GSK-3 $\beta$.

Mimuro et al (5) reported that treatment with DEX after myocardial ischemia in an isolated perfused heart preparation did not have a beneficial effect on infarct size, DEX induced an increase in infarct size compared with the reduction in infarct size observed in the present study. However, the preparations used in the two studies differed: In vitro in the previous study compared with in vivo in the present study. Furthermore, a previous study reported that the cardioprotective effects of morphine preconditioning on doxorubicin-induced heart 
failure were associated with the extracellular signal-regulated kinase/GSK-3 $\beta$ pathway, independent of PI3K/Akt (18). In the previous study, morphine preconditioning reduced infarct size and LDH activity caused by I/R injury, in accordance with the results of the present study. However, in the previous study, the protective effect of morphine preconditioning was abolished by extracellular-regulated kinase inhibition (PD98059), but not by PI3K inhibition (wortmannin). The current study also used wortmannin to block PI3K demonstrating that the protective effects of DEX on heart injury were dependent on PI3K/Akt signaling pathway.

Since DEX is a selective $\alpha-2$ adrenergic agonist, the cardioprotective effects observed in the present study may be due to activation of the $\alpha-2$ adrenergic receptor. Therefore, further studies are required to delineate the role of the receptor. In summary, DEX postconditioning exhibited protective effects on myocardial ischemia reperfusion injury and the effects may be dependent on the PI3K/Akt signaling pathway. The results of the current study may potentially provide a basis the use of DEX as a clinical therapy for cardiac I/R injury.

\section{Acknowledgements}

The present study was supported by the Anhui Educational Committee (grant no. KJ2012Z246), China. The authors would like to thank Professor Tak-Ming Wong (University of Hong Kong, Hong Kong, China) for reading and revising the manuscript.

\section{References}

1. Souter MJ, Rozet I, Ojemann JG, Souter KJ, Holmes MD, Lee L and Lam AM: Dexmedetomidine sedation during awake craniotomy for seizure resection: Effects on electrocorgraphy. J Neurosurg Anesthesiol 19: 38-44, 2007.

2. Ard J, Doyle W and Bekker A: Awake craniotomy with dexmedetomidine in pediatric patients. J Neurosurg Anesthesiol 15: 263-266, 2003

3. Cai Y, Xu H, Yan J, Zhang L and Lu Y: Molecular targets and mechanism of action of dexmedetomidine in treatment of ischemia/reperfusion injury. Mol Med Rep 9: 1542-1550, 2014.

4. Ibacache M, Sanchez G, Pedrozo Z, Galvez F, Humeres C, Echevarria G, Duaso J, Hassi M, Garcia L, Díaz-Araya G and Lavandero S: Dexmedetomidine preconditioning activates pro-survival kinases and attenuates regional ischemia/reperfusion injury in rat heart. Biochim Biophys Acta 1822: 537-545, 2012.
5. Mimuro S, Katoh T, Suzuki A, Yu S, Adachi YU, Uraoka M, Sano $\mathrm{H}$ and Sato S: Deterioration of myocardial injury due to dexmedetomidine administration after myocardial ischaemia. Resuscitation 81: 1714-1717, 2010.

6. Wetzker R and Rommel C: Phosphoinositide 3-kinases as targets for therapeutic intervention. Curr Pharm Des 10: 1915-1922, 2004.

7. Juhaszova M, Zorov DB, Yaniv Y, Nuss HB, Wang S and Sollott SJ: Role of glycogen synthase kinase-3beta in cardioprotection. Circ Res 104: 1240-1252, 2009.

8. Zhou PY, Zhang Z, Guo YL, Xiao ZZ, Zhu P, Mai MJ and Zheng SY: Protective effect of antiapoptosis potency of prolonged preservation by desiccation using high-pressure carbon monoxide on isolated rabbit hearts. Transplant Proc 47: 2746-2751, 2015

9. Dahlin LG, Kågedal B, Nylander E, Olin C, Rutberg H and Svedjeholm R: Early identification of permanentmyocrdial damage after coronary surgery is aided by repeated measurements of CK-MB. Stand Cardiovasc J 36: 35-40, 2002.

10. Yang C, Wu K, Li SH and You Q: Protective effect of curcumin against cardiac dysfunction in sepsis rats. Pharm Biol 51: 482-487, 2013.

11. Abdelrahman RS, El-Awady MS, Nader MA and Ammar EM: Hydrogen sulfide ameliorates cardiovascular dysfunction induced by cecal ligation and puncture in rats. Hum Exp Toxicol 34: 953-964, 2015.

12. Ran X, Diao JX, Sun XG, Wang M, An H, Huang GQ, Zhao XS, Ma WX, Zhou FH, Yang YG and Miao CM: Huangzhi oral liquid prevents arrhythmias by upregulating caspase- 3 and apoptosis network proteins in myocardial ischemia-reperfusion injury in rats. Evid Based Complement Alternat Med 2015: 518926, 2015.

13. Wong WW and Puthalakath $\mathrm{H}$ : Bcl-2 family proteins: The sentinels of the mitochondrial apoptosis pathway. IUBMB Life 60: 390-397, 2008

14. Brooks $\mathrm{C}$ and Dong Z: Regulation of mitochondrial morphological dynamics during apoptosis by Bcl-2 family proteins: A key in Bak? Cell Cycle 6: 3043-3047, 2007.

15. Lempiäinen J,Finckenberg P, Mervaala EE, Storvik M, Kaivola J, Lindstedt K, Levijoki J and Mervaala EM: Dexmedetomidine preconditioning ameliorates kidney ischemia-reperfusion injury. Pharmacol Res Perspect 2: e00045, 2014.

16. Yu Y, Jia XJ, Zong QF, Zhang GJ, Ye HW, Hu J, Gao Q and Guan SD: Remote ischemic postconditioning protects the heart by upregulating ALDH2 expression levels through the PI3K/Akt signaling pathway. Mol Med Rep 10: 536-542, 2014.

17. Zhou H, Hou SZ, Luo P, Zeng B, Wang JR, Wong YF, Jiang ZH and Liu L: Ginseng protects rodent hearts from acute myocardial ischemia reperfusion injury through GR/ER-activated RISK pathway in an endothelial NOS-dependent mechanism. J Ethnopharmacol 135: 287-298, 2011.

18. He SF, Jin SY, Wu H, Wang B, Wu YX, Zhang SJ, Irwin MG, Wong TM and Zhang Y: Morphine preconditioning confers cardioprotection in doxorubicin-induced failing rat hearts via ERK/GSK-3 $\beta$ pathway independent of PI3K/Akt. Toxicol Appl Pharmacol 288: 349-358, 2015. 